\title{
Scenario of Rectal Carcinoma Cases in a Rural Setting of Central India
}

\author{
Darshana Tote ${ }^{1}$, Rajesh Domakunti ${ }^{2}$, Sachin Tote $^{3}$ \\ 1,2 Department of Surgery, Jawaharlal Nehru Medical College, Sawangi, (Meghe), Wardha, Maharashtra, India. \\ ${ }^{3}$ Department of Anatomy, Jawaharlal Nehru Medical College, Sawangi, (Meghe), Wardha, Maharashtra, India.
}

\section{ABSTRACT}

\section{BACKGROUND}

Rectal cancer ranks second in the list of common cancers (28\%) in large intestine. Curative surgical resection is the treatment of choice for patients of carcinoma rectum. Very few studies on colorectal cancers are seen in Indian literature and the reason for this is the small number of patients reporting for treatment. Thus, to analyse demographic spectrum of rectal carcinoma in central India, this study has been undertaken.

\section{METHODS}

Audit was performed retrospectively to evaluate demography and management of rectal cancers in surgical in-patient department at single center in central India.

\section{RESULTS}

Males are more affected with predominance in the age group 40 to 60 years. Adenocarcinoma was the most common variant (86.6\%). $46 \%$ presented late hence could not be operated and required neo-adjuvant therapy.

\section{CONCLUSIONS}

Rectal carcinoma is a silent presenter and hence late manifestations are common. Good results can be achieved if multimodality treatment protocols are implemented.

\section{KEY WORDS}

Carcinoma Rectum, Treatment, Mortality
Corresponding Author: Dr. Darshana Tote, Department of Surgery, Jawaharlal Nehru Medical College, Sawangi, (Meghe), Wardha,

Maharashtra, India.

E-mail: drtotedarshana@gmail.com

DOI: $10.14260 / \mathrm{jemds} / 2020 / 753$

How to Cite This Article:

Tote D, Domakunti R, Tote S. Scenario of rectal carcinoma cases in a rural setting of central India. J Evolution Med Dent Sci 2020;9(46):3434-3438, DOI: 10.14260/jemds/2020/753

Submission 25-07-2020,

Peer Review 02-10-2020,

Acceptance 09-10-2020,

Published 16-11-2020.

Copyright (C) 2020 Darshana Tote et al. This is an open access article distributed under Creative Commons Attribution License [Attribution 4.0 International (CC BY 4.0)] 


\section{BACKGROUND}

Malignancy is the most threatening illness of our times. Many researches are going on in this field to find out the cause and treatment for the same. Cancer is a leading cause of death worldwide. The main reason behind this, is the delayed presentation and less awareness among the population regarding its presenting features. Colo-rectal cancers are a major health problem all over the world, leading the list at second position among the most common cancers. Rectal cancer is the most common malignant neoplasm seen in humans and ranks second in the list of common cancers $(28$ $\%)$ in large intestine after proximal colon (42\%). ${ }^{1}$ The screening programs are helping in early identification of the lesions thus helping in decreasing the incidence of rectal cancers but it is also noticed that young population is getting more affected by this pathology. ${ }^{2}$ Screening helps in evaluating the people at community level before onset of any symptoms and thus is very useful in early detection of disease and helpful in early intervening for definitive treatment. With the study of this magnitude there has been recent need of setting the priority, planning the programmes as per needs, development of policies within the country and allocations of the available resources. ${ }^{3}$ The definition of rectal cancer varies, but the most accepted definition is that carcinoma lying within $15 \mathrm{~cm}$ of the anal verge as measured by rigid proctoscopy. The rectum is commonly subdivided into three parts-upper $(>10-15 \mathrm{~cm})$, middle $(>5-10 \mathrm{~cm})$ \& lower $(0-5 \mathrm{~cm}) .{ }^{4}$ Every part of rectum has got its own incidence for occurrence of malignancy. Western lifestyle like less physical activity, high fat diet is largely showing predominance for this disease. ${ }^{5}$ Presentation of rectal cancer varies due to its location in deep pelvis. Also, its vicinity to genitor-urinary system affects the presentation of colorectal malignancy cases. ${ }^{1}$ As per the presentation the workup of patient differs so mainly it is diagnosed on colonoscopy and biopsy. ${ }^{5}$ Emergence of new diagnostic tools have enhanced the diagnosing rate of this condition. Newer imaging techniques and colonoscopy has helped a lot in early diagnosis of these lesions. Multimodality treatment helps in increasing the survival rates of these patients. ${ }^{1}$ the most common cellular type in colorectal carcinomas is adenocarcinoma. ${ }^{5}$ Complete surgical resection of tumour is the definitive treatment for these patients. The newer modality like Total Mesorectal Excision (TME) along with neo-adjuvant chemo radiotherapy shows improved survival rates due to good local control. ${ }^{6}$ Inclusion of radiotherapy has further added the benefit of long term survival. ${ }^{7}$ The oncologists in India believe that most cases of colorectal cancers presenting in younger age groups, has more advanced variety with more signet ring morphology, and more anorectal as compared to colonic site of primary as compared to that reported worldwide. But due to lack of adequate supportive literature reaching to any conclusion regarding such presentation in Indian population is difficult. Indian literature shows very few studies on colorectal carcinoma that too with a less study population. Moreover, there are very few studies on demography and presenting symptoms of colorectal cancers patients. Hence, the profile of patients with colorectal cancers in India is unknown. ${ }^{8}$ This study was undertaken with an aim to analyse the demographic spectrum of rectal carcinoma in central India.

\section{METHODS}

This is a retrospective study carried out at a multispecialty tertiary care hospital situated in central India. IEC (Institutional Ethical Clearance) was sought for the study. The aim of this study was to evaluate the demography, clinical presentation and type of management that rectal carcinoma patients are subjected to and compare it with related literature that are available in India as well as Western literature. All patients diagnosed or suspected with rectal carcinoma on basis of presentation, imaging and tissue diagnosis who presented to the centre were evaluated in the Surgical InPatient Department by a multidisciplinary team including a general surgeon, medical oncologist, radiologist \& pathologist. A prospective database included demographic features, symptoms, primary diagnosis, basic laboratory investigations, staging and treatment given. All the patients diagnosed with rectal carcinoma in the last three years $(2017$ - 2019) in this single centre were included in this study. The diagnosed cases were graded as; Grade I: Well differentiated, Grade II: Moderately differentiated and Grade III: Poorly differentiated. Statistical analysis was done by using percentage analysis method.

\section{RESULTS}

A total of 30 cases of rectal carcinoma patients was enrolled during the study period as per the available database. The study population showed male predominance with $63.4 \%$ of total study group while female group comprised of $36.6 \%$. Age of the patient in present study database varied from 25 years to 87 years with mean of 51.1 yrs. (with standard deviation of \pm 15.7). Maximum number of patients were seen between the age group of 40 to 49 years i.e., 8 patients (26.60 \%) followed by $23.4 \%$ in age group $50-59$ years and $16.6 \%$ in $60-69$ years group. The least number of patients i.e., zero (0 \%) in the age group below 20 years and above 90 years.

On categorizing the patients as per presenting complaints majority of the patients (83.40\%) presented with bleeding per rectum as their chief complaint followed by altered bowel habits and pain in abdomen in $33.40 \%$ and $16.70 \%$ of total patients respectively. This study population was evaluated on the basis of histological type of the growth which showed that adenocarcinoma was the leading histological type seen in $86.60 \%$ of the total cases other types detected were mucinous adenocarcinoma, signet ring cell type carcinoma etc in 3,1 and nil patients of the study respectively. (Table 1) On further categorising the patients as per histological grading, grade I was present in $60 \%$ of total cases followed by grade ii and grade III with $23.50 \%$ and $16.40 \%$ of total cases respectively. (Graph 1)

In the study population $46.80 \%$ of patients were nonoperable, hence subjected to neo-adjuvant chemo-radiation. $33.20 \%$ of patients underwent abdomino-perineal resection with permanent colostomy, $16.60 \%$ of patients underwent anterior resection \& $3.40 \%$ of patients underwent division colostomy only (as they were in-operable but presented with obstruction and had to be subjected to palliative line of management) as depicted in table 2 


\begin{tabular}{|ccc|}
\hline Histological Type & No. of Patients & Percentage (\%) \\
Adenocarcinoma & 26 & 86.60 \\
Mucinous Adenocarcinoma & 3 & 10 \\
Signet ring cell type & 1 & 3.40 \\
Others & 0 & 0 \\
Total & $\mathbf{3 0}$ & $\mathbf{1 0 0}$ \\
\hline Table 1. Distribution of Patients Based on Their Histological Type \\
\hline
\end{tabular}

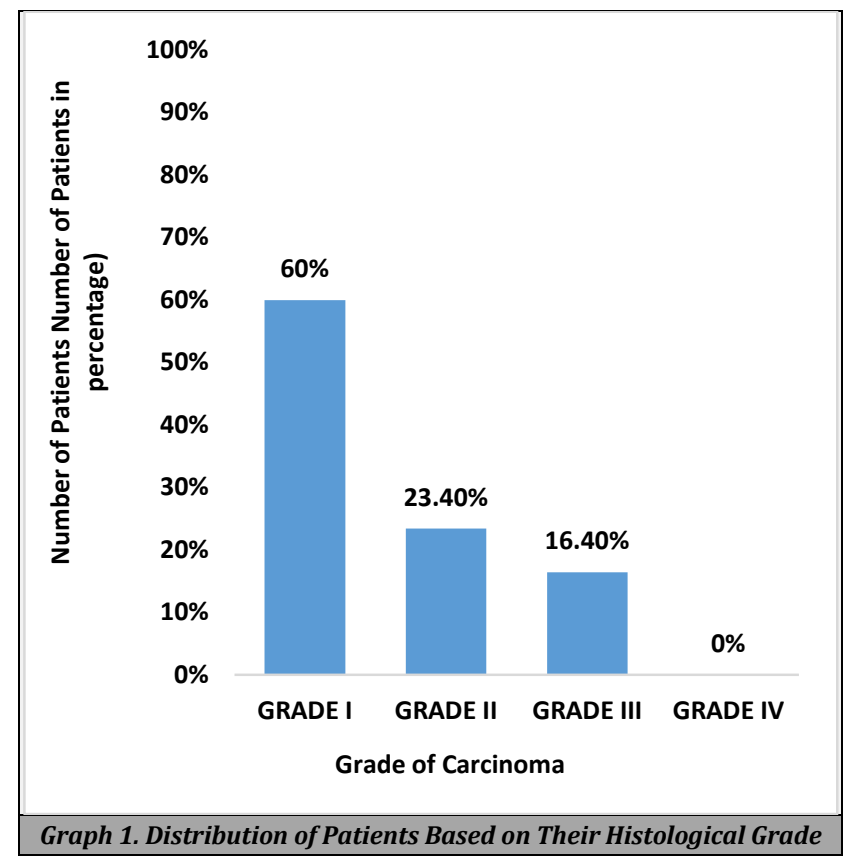

\begin{tabular}{|ccc|}
\hline Type of Surgery & No. of Patients & Percentage \\
Anterior Resection & 5 & 16.60 \\
Abdomino-perineal Resection & 10 & 33.20 \\
Colostomy only & 1 & 2.40 \\
No surgery & 14 & 46.80 \\
Total & $\mathbf{3 0}$ & $\mathbf{1 0 0}$ \\
\hline Table 2. Distribution of Patient Based on The Type of Surgery \\
\hline
\end{tabular}

\begin{tabular}{|ccc|}
\hline Chemo-Radiation & No. of Patients & Percentage \\
Neo-Adjuvant & 16 & 53 \\
Adjuvant & 14 & 47 \\
Total & 30 & 100 \\
\hline \multicolumn{2}{|c|}{ Table 3. Distribution of Patients } \\
Based on the Type of Chemo-Radiation \\
\hline
\end{tabular}

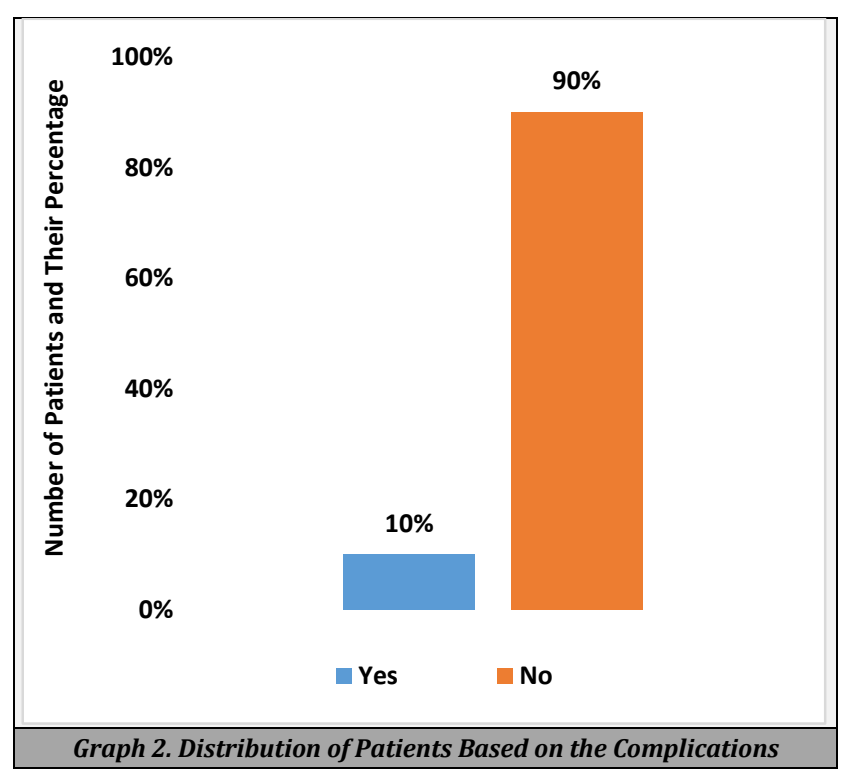

Out of 30 patients taken into the study, $53 \%$ of patients underwent neo-adjuvant chemo-radiation \& remaining $47 \%$ of patients underwent adjuvant chemoradiation as supportive management. (Table 3 ). Only $10 \%$ of the patients developed complications due to one of the treatment modalities (surgery / chemotherapy / radiotherapy). (Graph 2)

None of the patients showed evidence of recurrence after management of rectal carcinoma in our study after 6 months of follow up. Reasons for which may be many like lost to follow up or fear for any modality of treatment or just negligence regarding further supportive treatment after definitive treatment was done.

\section{DISCUSSION}

Malignancy is one of the leading killers of modern society due to modern lifestyle and diet modifications. Carcinoma rectum is the second most common site for cancer in large intestine after proximal intestine. ${ }^{1}$ Incidence of rectal carcinoma in Western countries and India are 12 - 14 and 4 - 5 per 1 lakh population respectively. Rectal carcinoma is listed as most frequently diagnosed type of gastro-intestinal carcinomas with high incidence of disease related mortality. On the contrary the Indian scenario is quite different. There are low incidences of rectal carcinoma but mortality is too high due to delayed diagnosis and referral from peripheral parts of country. ${ }^{9}$ As it is one of the leading cancer worldwide causing greater problem to human healthcare system, study of demographic profile of these cancer cases will help us to identify the various loopholes in the medical science related to rectal carcinoma, which will ultimately guide everyone to an advanced way of screening, diagnosing and treating the patient and thus tackle the burden of the disease.

Incidence of rectal carcinoma are higher for men compared to women worldwide. $83.40 \%$ of the patients in our study were males with male to female ratio of 1.72:1. The mean age of the patients is 51.1 years with a standard deviation of 15.7 in our study. $26.60 \%$ of the patients were between 40 - 49 years of age. A similar study in India was done by Prachi S Patil et al, in which $65 \%$ of patients were males. Mean age of the patients was 47.2 years. Most of the patients (44\%) were in the age range of $40-60$ years. ${ }^{8}$ As per the study done in Kashmir, India by Dar Abdul Waheed et al, male to female ratio was 1.25: 1 . Most common age group is $<45$ years. This study depicted Jung-A Yun et al study which revealed male preponderance $60.1 \%$ with median age of 59 years. ${ }^{11}$ Study done by Suryanarayana Deo et al showed $68.50 \%$ of male patients. 45.4 years was the mean age of the study population. Study mentioned that incidence of rectal carcinoma in Western countries varied from 12 - 14 / 100000 population while in Indian continent it is $4-5 / 100000$ population. Mean age of rectal cancer patients in western world is 60 - 70 years while in their study mean age was 45.4 years. ${ }^{9}$ Curtis Mettlin et al conducted a study in United States, showing increased incidence in males constituting $54.60 \%$ of total patients taken in study. Maximum number of patients were between the age range of 50 - 69 years (52.75\%).12 Another study conducted in United States by Nestor F Esnaola et al, average age of patients was 65 years and percentage of cases in males was 59.6 of total patients taken in study. ${ }^{13}$

Based upon several studies it reveals that rectal carcinoma is more common in males and younger age group in India as 
compared to western countries like United States where it is more common in males and older age group.

Patients of rectal carcinoma do not present with any chief complaints until the tumour extends into the adjacent organs or into the lumen of gastro-intestinal tract. There has been increase in the screening programs for rectal carcinoma also. Hence, most of the patients are diagnosed during such screening and their presentation is asymptomatic. But if the patients present with the chief complaints, they are per rectal bleeding, altered bowel habits and abdominal pain. ${ }^{1}$ In our study most of the patients presented with complaints of per rectal bleeding ( $83.40 \%$ ). Some of the patients had complaints of altered bowel habits (33.40 \%) and abdominal pain (16.70 $\%)$. Study of Prachi S Patel et al, in Indian scenario shows that patients of rectal carcinoma presented with chief complaints of rectal bleeding, abdominal pain \& altered bowel habits with $56.5 \%, 44 \%$ and $25.6 \%$ respectively. ${ }^{8}$ A similar study was done by Satyanarayana Deo et al, which revealed that $88 \%$ of the patients taken in study presented with complaints of rectal bleeding followed by altered bowel habits and abdominal pain with $47 \%$ and $32 \%$ respectively. ${ }^{9}$

Biopsy is the gold standard technique to diagnose the rectal carcinoma. The biopsy specimen has to be carefully examined for a proper tissue diagnosis. The pathologist has to differentiate the tissue from various premalignant lesions, scarring diverticulitis and solitary rectal ulcers which occur during rectal prolapse. Most common histologic type found in rectal carcinoma is adenocarcinoma, identified by epithelial columnar cells arranged in glandular pattern. The prognostic marker for this malignancy is grade of tumour which is assessed during the pathological typing on basis of number of glandular structures observed. Grading of carcinoma on the basis of level of differentiation of glandular structures developed in rectal mucosa will predict the prognosis of the patient. ${ }^{2}$ Mucinous and signet ring cell histology are less common variants. ${ }^{6}$ In our study most common histologic variant seen was adenocarcinoma (86.60\%) followed by mucinous (10\%) and signet ring cell (3.4\%) respectively. Grading was done to access prognosis. $60 \%$ of the patients had grade I (well differentiated) followed by grade II $(23.40 \%)$ and grade III (16.40) respectively.

In our study, out of 30 patients, $46.80 \%$ of patients were non operable, $33.20 \%$ of patients underwent abdominoperineal resection, $16.60 \%$ of patients underwent anterior resection \& $3.40 \%$ of patients underwent colostomy only. Similarly, in a study done by Deo S et al in $200475.2 \%$ were operable and underwent curative surgery whereas $24 \%$ underwent palliative colostomy after exploration as the lesion was found to be non-operable. The study stated that radical surgery is the surgery of choice but currently radiotherapy has also become an important part of treatment. It enhances the loco-regional control. It can be given pre or post operatively. It has got advantage as it can be given post operatively when the tumour burden is reduced. But it carries with it the drawbacks of gastro-intestinal tract adhesions. Most European studies prefer pre-operative radiation rather than post-operative radiation as it helps in down staging of tumour and sterilization of surgical margins. Moreover, as the radiation is given to well oxygenated tumour cells the response to radiation is good. Whereas the Swedish trial suggests that short course preoperative regimen of radiotherapy is highly useful in loco-regional control of disease preoperatively. ${ }^{9}$
In our study, out of 30 patients, 16 patients (53\%) underwent neo-adjuvant chemo-radiation \& 14 patients (47 $\%$ ) underwent adjuvant chemo radiation. As per study done by Jung-A Yun et al, out of 2050 patient taken in the study, 356 patients $(17.40 \%)$ underwent neo-adjuvant chemo-radiation and $1694(82.60 \%)$ patients underwent adjuvant chemoradiation. The study states that pre-operative concomitant chemoradiation do not lower the local recurrence rate but it significantly reduces the distant and local recurrence rate. The neo adjuvant chemoradiation has shown complete pathological response, tumour down staging and improved sphincter preservation with local control in cases of locally advanced rectal cancers. 11

In our study, out of 30 patients, only $10 \%$ of patients developed complications whereas $90 \%$ of patients developed no complications after management of rectal carcinoma. Whereas in a study done by Deo S et al $200423.5 \%$ cases showed post-operative morbidity like wound infection, prolonged ileus, haemorrhage and wound dehiscence..$^{9}$

\section{CONCLUSIONS}

Rectal carcinoma is a silent presenter and hence late manifestations are common. Its presentation and outcome in Indian scenario are different from western population. There is a need to indulge in more literature-based studies for Indian population to evaluate the current situation of disease in our population. Introduction of a programmed screening schedule is needed for early detection of these cases; otherwise, they land up in colostomy which itself is a not an easily accepted procedure in the general population. Good results can be achieved if multimodality treatment protocols are implemented by well trained specialists from all fields related to malignancy.

Data sharing statement provided by the authors is available with the full text of this article at jemds.com.

Financial or other competing interests: None.

Disclosure forms provided by the authors are available with the full text of this article at jemds.com.

\section{REFERENCES}

[1] Fazeli MS, Keramati MR. Rectal cancer: a review. Med J Islam Repub Iran 2015;29:171.

[2] Goldenberg BA, Holliday EB, Helewa RM, et al. Rectal cancer in 2018: a primer for the gastroenterologist. Am J Gastroenterol 2018;113(12):1763-71.

[3] Tardivo S, Mantovani W, Torri E, et al. Rectal cancer. Epidemiology and burden of disease. In: Delaini GG, edr. Rectal Cancer. Springer 2005:1-21.

[4] Bondeven P. Cancer of the upper rectum. Dan Med J 2016;63(10):B5227.

[5] Tumwine LK, Kagimu M, Ocama P, et al. Atypical presentation of colon adenocarcinoma: a case report. J Med Case Rep 2012;6:58.

[6] Gaertner WB, Kwaan MR, Madoff RD, et al. Rectal cancer: an evidence-based update for primary care providers. World J Gastroenterol 2015;21(25):7659-71. 
[7] Feeney G, Sehgal R, Sheehan M, et al. Neoadjuvant radiotherapy for rectal cancer management. World J Gastroenterol 2019;25(33):4850-69.

[8] Patil PS, Saklani A, Gambhire P, et al. Colorectal cancer in India: an audit from a tertiary center in a low prevalence area. Indian J Surg Oncol 2017;8(4):484-90.

[9] Deo S, Kumar S, Shukla N, et al. Patient profile and treatment outcome of rectal cancer patients treated with multimodality therapy at a regional cancer center. Indian J Cancer 2004;41(3):120-4.

[10] Waheed DA, Ahmad DS, Ahmad So, et al. Demographic profile of cancer colorectal and anal canal, a study conducted government medical college Srinagar Kashmir India: a retrospective study. Int J Surg Sci 2019;3(1):3547.
[11] Yun JA, Huh JW, Kim HC, et al. Local recurrence after curative resection for rectal carcinoma: the role of surgical resection. Medicine (Baltimore) 2016;95(27):e3942.

[12] Mettlin C, Natarajan N, Mittelman A, et al. Management and survival of adenocarcinoma of the rectum in the United States: results of a national survey by the American College of Surgeons. Oncology 1982;39(5):26573.

[13] Esnaola NF, Stewart AK, Feig BW, et al. Age-, race-, and ethnicity related differences in the treatment of nonmetastatic rectal cancer: a patterns of care study from the national cancer data base. Ann Surg Oncol 2008;15(11):3036-47. 